\title{
NMR LIPIDOMICS OF ANIMAL MODEL FOR SCHIZOPHRENIA
}

\author{
Raniery W. Ricardo (IC), Banny S. Barbosa (PG), Ljubica Tasic (PQ).
}

\begin{abstract}
Schizophrenia is a very severe and uncurable brain illness that has affected people throughout history. Medical treatments focus on eliminating the symptoms of this illness and count on use of different antipsychotic medications. It is assumed that this illness can be caused by several factors among which stands altered brain chemistry. This work aims to correlate the composition of lipids isolated from the serum samples of animals used as model animals for schizophrenia and also reveal the antipsychotic drug effects on lipidomics. For these, four groups of animals (Wistar mice) were used: healthy, drug-treated healthy, schizophrenia model and drug-treated schizophrenia model. Total lipids were extracted and analyzed with Nuclear Magnetic Resonance ( ${ }^{1} \mathrm{H}$ NMR) and chemometrics.
\end{abstract}

Key words: schizophrena, lipids, nuclear magnetic resonance.

\section{Introduction}

Schizophrenia is very severe mental disorder of the central nervous system (CNS), which affects $1 \%$ of world population. It is characterized with the absence of contact with reality, also deliriums and hallucination. There is still lack of information regarding its probable cause, and the brain's chemistry disbalance is one of the hypotesis. Alteration on lipid or sugar metabolism can contribute significantly to this illness. This work presents our recent research results on differences in lipidomics of animal model for schizophrenia, treated with different antipsychotic drugs already used in medical treatment of this illness.

\section{Results and Discussion}

Animal serum samples were carefully collected and prepared by our collaborators under lead of professor Hayashi (UNIFESP, SP). These were transported and kept for not more then 14 days at $-80^{\circ} \mathrm{C}$ until analyzed. Overall, four types of animal serum samples: (1) healthy animals, (2) animals used as model for schizophrenia, (3) treated healthy animals and (4) treated animals - model for schizophrenia, were analyzed.

The extracts of total lipids from serum samples were obtained using somewhat modified method published in 2008 by Tukiainen et al. ${ }^{1}$. Samples had $1.4 \%$ (healthy), $2.1 \%$ (schizophrenia model, SCZ), $1.3 \%$ (healthy drug-treated) and $1.6 \%$ (SCZ drug-treated).

The ${ }^{1} \mathrm{H}$ NMR spectra were recorded (Bruker, 600 $\mathrm{MHz})$ from $\mathrm{CDCl}_{3}(500 \mu \mathrm{L})$ sample $(5 \mathrm{mg})$ solutions. Lipids, like triacylglycerols, fatty acids with medium chains, and polar lipids have been identified based on the spectral data (Figure 1). Applying the Principal Component Analysis (PCA), we were able to evidence separation between healthy and SCZ samples due to the differences in PC2 scores. Also, drug-treated SCZ samples from the SCZ due to the differences in the PC1 scores. NMR spectral regions from 0.00 to $2.00 \mathrm{ppm}$ and from 6.00 to $10.00 \mathrm{ppm}$ were the most important for separations of the analyzed samples in healthy, SCZ, and SCZ drug-treated.

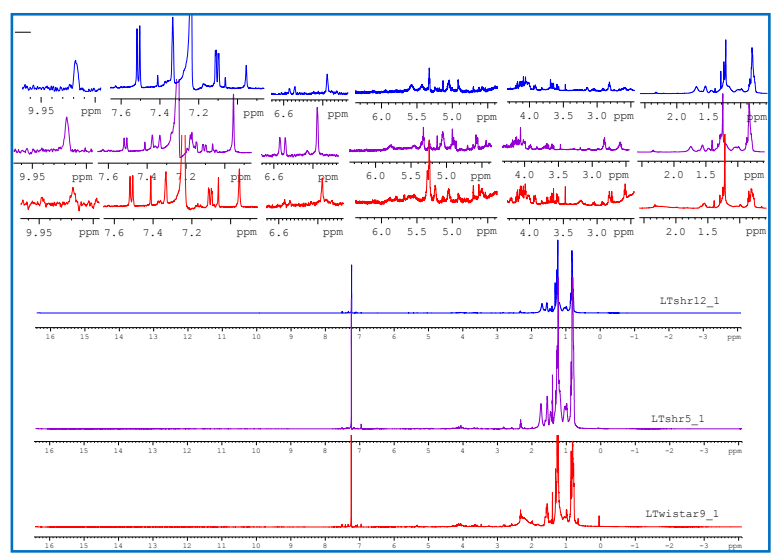

Figure 1. Comparison of the ${ }^{1} \mathrm{H}$ NMR spectra of total lipids from the serum of healthy (red), SCZ (purple) and SCZ drug-treated (blue).

\section{Conclusions}

The lipidomics by NMR applied on animal models for schizophrenia shown that used methodology can bring new insights on metabolic alterations on lipids and apolar compounds provoked by antipsychotic drugs. Also, it was possible to notice drug effects on the lipidomics of the following groups: healthy and drug-treated, and SCZ model and SCZ drug-treated.

\section{Acknowledgement}

FAPESP (2014/18938-8), CNPq (454234/2014-7) Substantiated opinion of the Ethics Commitee Code: 842.072

${ }^{1}$ Tukiainen, T. et al. Biochem. Biophys. Res. Commun. 2008, 375, 356-361. 